\title{
Computational modelling for cerebral aneurysms: risk evaluation and interventional planning
}

\author{
${ }^{1}$ Y VENTIKOS, PhD, ${ }^{1}$ E C HOLLAND, MEng, ${ }^{1} \mathrm{~T} J$ BOWKER, MEng, ${ }^{1} \mathrm{P}$ N WATTON, PhD, ${ }^{1} \mathrm{~N}$ M P KAKALIS, PhD, \\ ${ }^{2} \mathrm{M}$ MEGAHED, Dr.-Ing, ${ }^{2} \mathrm{~F}$ ZHU, PhD, ${ }^{3} \mathrm{P}$ E SUMMERS, PhD and ${ }^{3} \mathrm{~J} V \mathrm{BYRNE}, \mathrm{MD}$ FRCR
}

\begin{abstract}
${ }^{1}$ Fluidics and Biocomplexity Group, Institute of Biomedical Engineering and Department of Engineering Science, University of Oxford, Oxford, UK, ${ }^{2}$ ESI Group, Paris, France and ${ }^{3}$ Department of Neuroradiology, Nuffield Department of Surgery \& John Radcliffe Hospital, University of Oxford, Oxford, UK
\end{abstract}

\begin{abstract}
Advanced computational techniques offer a new array of capabilities in the healthcare provision for cerebral aneurysms. In this paper information is provided on specific simulation methodologies that address some of the unanswered questions about intracranial aneurysm and their treatment. These include the evaluation of rupture risk, the thrombogenic characteristics of specific lesions and the efficacy assessment of particular interventional techniques and devices (e.g. endovascular coil embolisation and flow diversion using stents). The issues connected with ease-of-use and interactivity of computed simulations is discussed, and it is concluded, that the potential of these techniques to optimise planning of complex and multifaceted interventions is very significant, in spite of the fact that most of the methodologies described are still being developed and perfected.
\end{abstract}

DOI: $10.1259 /$ bjr/14303482

(C) 2009 The British Institute of Radiology
As a result of increasing numbers of diagnostic brain scans, more intracranial aneurysms are being incidentally detected. Their diagnosis and the potentially dire consequences of rupture create a dilemma for patients and doctors, a dilemma centred on the risk of aneurysms growing or bleeding and amplified by the availability of minimally invasive endovascular treatments. Estimates of the incidence of aneurysmal subarachnoid haemorrhage range from 9 to 20 per 100000 [1], considerably lower than aneurysm prevalence (3.6\% at autopsy, $6.0 \%$ via angiography [2]). Thus, the majority of aneurysms do not rupture, a consistent finding in observation studies $[3,4]$. For the individual diagnosed with an unruptured aneurysm the uncertainty remains and, despite the statistical reassurance, substantial numbers request prophylactic (and potentially unnecessary) surgical clipping or coiling.

Computational studies are now being applied to this problem in order to understand the mechanisms that cause aneurysm formation, growth and rupture. There have been many previous attempts to explain why the particular arteries forming the circle of Willis are liable to develop aneurysms and it is generally agreed that anatomical, genetic, haemodynamic and pathological factors are involved [5]. Understanding their relative contributions and applying this knowledge to the individual seeking advice after diagnosis can be considered a mechanical as well as a biological challenge. Patient-specific computational simulations offer a solution to evaluate the risk of rupture objectively and are equally pertinent to treatment planning and follow-up assessments.

Address correspondence to: James V Byrne, Department of Neuroradiology, Nuffield Department of Surgery \& John Radcliffe Hospital. E-mail: James.Byrne@nds.ox.ac.uk
Aneurysm rupture occurs when stress exceeds the strength of the wall tissue. Accurate mechanical models, which can predict the stress distributions in an aneurysm, have the potential to assist diagnostic decisions [6]. However, the micro-structure, material properties, thickness and the strength of the aneurysm tissue are difficult to determine non-invasively. Consequently, there is uncertainty in the advantages of using a stress-based criterion for rupture; statistical trials are required to see if they are more effective than criterions based on size or growth rates. To develop improved mechanical models of the tissue structure, data is needed from histological studies in order to understand how it evolves. Computational models that simulate the adaptive growth and modelling of the tissue and evolution of an aneurysm may yield insight into the aetiology of the disease and may ultimately be able to predict an aneurysm's evolution/rupture. It is important to note that the cells that remodel the tissue are influenced by local mechanical stimuli, e.g. cyclic stretching and wall shear stress due to the blood flow $[7,8]$. To predict aneurysm evolution, it is necessary to develop sophisticated computational models that can model the fluid dynamics within the aneurysm, the mechanical properties of the tissue and the functionality of the cells within the arterial wall [9]. To this aim, conceptual models of aneurysm growth are being developed, e.g. where elastin degradation is linked to the local haemodynamics and collagen synthesis is linked to local stretching of the fibroblast cells $[10,11]$. These provide the basis for more realistic models of aneurysmal disease, and will ultimately be extended to physiological geometries.

Once the need to intervene has been established, computational simulation techniques are proving capable of evaluating the efficacy of treatment. For example, Jou et al [12] studied the haemodynamics within a 
fusiform basilar artery aneurysm, before and after the simulated occlusion of one vertebral artery, and Hassan et al [13] used computational fluid dynamics (CFD) techniques to simulate the haemodynamic effects of parent vessel occlusion for the treatment of a giant vertebro-basilar aneurysm. In terms of the coil embolisation method, Groden et al [14] simulated the coiling of an idealised geometric model, in which the coils were represented by blocked cells in the aneurysm lumen mesh. Byun and Rhee [15] reported similar calculations for artificially constructed models focusing on different parent vessel geometries and coil placement, assuming coils to be a sphere of blocked cells.

As an adjunct to coiling, it is now becoming common practice to promote endovascular occlusion of aneurysms by means of stenting. Aenis et al [16] and Stuhne and Steinman [17] both explored the haemodynamics in basic three-dimensional (3D) models of stented basilar side-wall aneurysms using CFD and finite element (FE)based CFD, respectively. Cebral and Lohner [18] took this one step further by running CFD simulations implementing an adaptive embedded mesh technique to patient-specific geometries and used virtual deployment of coils and stents. Similar conclusions are drawn in all three studies: the presence of a stent demonstrates a reduction in flow velocities into the aneurysm sac. Radaelli et al [19] presented similar haemodynamic simulation results for anatomically accurate stented aneurysms calculated independently by six international groups. Aneurysmal velocity magnitude and wall shear stress results illustrated the reproducibility of flow simulations and hence the increasing ability of current haemodynamic modelling technology to consistently quantify the performance of commercial intracranial stent implants in general.

Computational simulation techniques also offer a wider range of possibilities to simulate phenomena and mechanisms of interventions, and, in particular, the exploitation of thrombosis to stop blood flow into the aneurysm lumen. In the long term, the type of thrombus initiated through intervention may be of considerable importance. In vivo there are numerous damage mechanisms which may lead to thrombus formation [20, 21]. Tissue factor and collagen are considered two key players at initiation: expression of either of these proteins leads to platelet activation, which has proven necessary for a platelet to become stably adhered to the growing thrombus [22]. Tissue factor also initiates thrombin generation through the coagulation cascade, which amplifies its own production, activates platelets and leads to fibrin generation.

The understanding of the coagulation cascade has been enhanced by the extraction of rate parameters for individual reaction pathways: the computational work of Hockin et al [23] and, more recently, Panteleev et al [24] is particularly notable for the detail incorporated. When additionally accounting for the effect of convection, the complexity of the coagulation cascade necessitates innovative modelling approaches. Fogelson and Tania [25] utilised mass transport terms to account for the influence of flow upon the reaction pathway, whereas Guy et al [26] utilised methods traditionally employed to describe the gelation of polymers to investigate the limiting size of a fibrin gel in a shear flow. There is considerable value in investigating individual components of the thrombus, and in order to capture macroscopic detail (e.g. thrombus morphology) it is necessary to capture processes occurring on disparate scales. This review will present salient features of recently developed computational techniques that address these challenges.

\section{Methods and materials}

The common theme underlying the techniques presented in this paper is the transformation of the problems in question into a mathematical form and the efficient solution of the pertinent equations through techniques of computer simulation.

\section{Growth and remodelling}

A prerequisite to modelling the evolution of an aneurysm is to accurately model the healthy artery. In recent years, sophisticated theoretical models of arteries have been developed that model the mechanical response of each layer of the artery, the individual constituents, i.e. the elastin and collagen, and their structural arrangement [27]. Watton et al [28] adapted this constitutive model to introduce micro-structural aspects of the tissue so that the degradation of elastin and growth and remodelling of the collagen fabric could be simulated to model aneurysm progression. Evolution of clinically measurable parameters was consistent with experimental studies for a growth model of abdominal aortic aneurysms [29]. We follow this approach to simulate the evolution of saccular cerebral aneurysms of the internal carotid artery. Moreover, we combine the structural model of the artery with a haemodynamic analysis so that the growth and remodelling of the tissue can be explicitly linked to the haemodynamic environment [10].

The internal carotid artery is modelled as a cylindrical membrane subject to physiological stretches, i.e. an axial pre-stretch of 1.3 and circumferential stretch of 1.25 at systolic pressure $(16 \mathrm{kPa})$; the systolic radius is $2 \mathrm{~mm}$ and the length is $16 \mathrm{~mm}$. The elastinous constituents are initially assumed to bear $80 \%$ of the load of the arterial wall at systole. Initially, the geometry of the model of the artery is cylindrical. Consequently, the initial wall shear stress (WSS) distribution that acts on the luminal layer of the artery is constant. To achieve an altered haemodynamic environment, i.e. altered spatial WSS distribution, a small out-pouching of the arterial domain is created. The change to the geometry of the domain alters the spatial distribution of the WSS that acts on the endothelial lining. This allows us to explore hypotheses linking subsequent growth and evolution of the aneurysm to haemodynamic stimuli experienced by the endothelial cells. A pressure correction, finite volume solver for the haemodynamics CFX (ANSYS, Canonsburg PA) was coupled with an in-house finite element solver for the vascular wall dynamics, together with appropriate routines for the domain deformation, stimulus propagation and remeshing. 


\section{Interventional modelling}

This section first describes the steps undertaken to capture the flow diversion induced by the insertion of stents across the aneurysm neck and, subsequently, presents the method used to capture the insertion of coils within the aneurysm lumen.

All simulations were performed in CFD-ACE+ (ESI Group, Paris, France), which utilises a finite volume solver. A finite volume mesh is generated by dividing the region of interest into a number of control volumes (cells); the average value within each cell is stored at the cell centre and interpolation is used to describe variations away from the centroid. This enables the solution of the governing Navier-Stokes equations within arbitrarily complex geometries. The resulting mesh consists of a set of non-overlapping polyhedra that cover the entire region of interest. Complex geometries have traditionally been captured by utilising tetrahedron-only meshes; in this study, a different approach is taken, using an adaptive unstructured cartesian mesh generated using CFD-VisCART (ESI Group, Paris, France). In brief, the bulk volume is discretised with structured hexahedral elements. Near to the boundary, consisting of the aneurysm wall and stent struts, the mesh is refined by splitting the hexahedral elements to form deformed polyhedral cells that fit the desired volume of interest.

A principal advantage of this method is that it minimises the level of user interaction required in order to generate a mesh, potentially opening the door for incorporating flow analysis as a routine component of interventional planning. The geometric data made available for mesh generation are usually a collection of parts coming from various sources, e.g. geometry restructuring from MRI images and designed stent geometry. The full assembly typically includes a number of overlapping surfaces and unintended gaps. Even for the parts that are properly mated together, the mating surfaces have dissimilar surface discretisations; conventional mesh generators require a watertight surface mesh, with perfect node-to-node matching of the triangles that constitute the surface of the mating parts. Therefore, a significant amount of effort is required to transform (clean) the raw geometry data into a fully "connected" and closed set of surfaces.

In order to avoid geometry cleaning, the interior volume grid is generated first and then it is connected with the boundary. A schematic of this grid generation technique is shown in Figure 1 [30].

First the interior cartesian grid is generated, then the boundary is connected through projections. The connection of the interior grid to the boundary can be based on the minimum distance direction. In order to generate the volume grid, the user is requested to define a maximum and a minimum cell size. The only requirement that the set of geometric entities must satisfy is that the computational domain formed with the entities is "physically" closed if gaps or holes smaller than minimum cell size are ignored. Note that this enclosure condition is much weaker than the condition of "watertightness" required by traditional approaches. Obviously, if all the gaps between the line segments in Figure 1 are smaller than the minimum cell size, the set of line segments actually defines a valid computational domain.

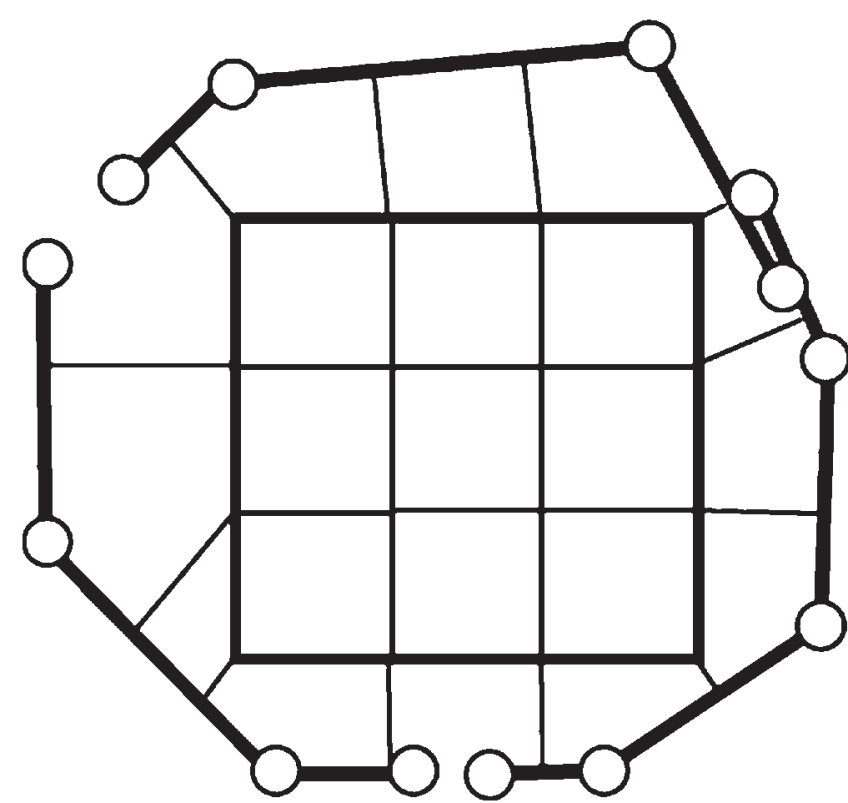

Figure 1. The automated VisCART grid generation technique; the first step involves the formation of a luminal grid, which is subsequently projected to the boundary.

Although the mesh generation technique described above is suitable for capturing the incorporation of a stent, for modelling aneurysms treated by endosaccular packing with coils, the computational requirements for directly resolving the entire length of the coil are still prohibitive. Therefore, alternative, indirect techniques are necessary. This paper presents novel computational methodologies for patient-specific modelling of endovascular coil embolisation, describing the haemodynamic behaviour of the combined vasculature-catheter system during the stages of coil introduction to an aneurysm sac. The anatomically accurate vasculature geometry is reconstructed by employing 3D rotational angiography data sets, and we model the coiled part of the aneurysm as a porous medium of decreasing porosity as coils are deployed. Hence, the aneurysm lumen is split in a pure fluid (blood) region corresponding to the coil-free part and a porous one corresponding to the actually coiled aneurysmal volume [31]. For the pure fluid region, the haemodynamics is described by the classical Navier-Stokes equations. For the porous part, these can be recast to also include the porosity and permeability of the porous medium and the drag factor across the porous flow. The porosity represents the volume occupied by the pores to the total volume of the system and is a function of the occluded aneurysm volume. The permeability is a measure of the fluid conductivity through the porous medium, representing the surface area to volume ratio of the porous matrix. Here, it is considered to be given by Kozeny's theory [32] and the calculations are based on the characteristics of the actual coils used in the intervention.

The porosity-based modelling offers consistency and grid independence benefits when compared with methodologies that describe the coils, either via blocked cells or through full resolution of the near-coil environment. Although the latter is an accurate and consistent 
approach, which has been used with success in modelling of open stents, the resolution requirements for capturing the significantly longer coil lengths render this approach prohibitively expensive. Such approaches converge to grid-independent solutions only when the fluid dynamics around the actual wire cross-section is resolved, which is impossible computationally for fully coiled aneurysms. The porosity approach, being of a statistically distributed nature, eliminates the uncertainty introduced by the level of local grid refinement around individual coils, and is, therefore, computationally efficient and self-consistent, as it can lead to gridindependent solutions.

\section{Multiphysics modelling: thrombosis}

In the work presented here, we discount the biochemical pathway and present an investigation of the effect of shear stress on thrombus morphology. The thrombus is modelled as a porous medium (with properties derived from Diamond [33]) and its growth across the domain is tracked through level sets. The level set method encodes the position of the thrombus surface in a signed distance function. The signed distance function is sampled upon a uniform grid, the value at each point being the euclidean distance to the surface with the caveat that it takes on a negative sign when a grid point is inside the thrombus and a positive sign when external to the thrombus. Platelet deposition can be accounted for by propagating the interface under a normal velocity field ( $\left.V_{\text {normal }}\right)$; this is achieved by solving a partial differential equation:

$$
\frac{\partial \phi}{\partial t}=V_{\text {normal }}|\nabla \phi|
$$

Porous-level set coupling is achieved by stipulating that the porosity field $(\varepsilon)$ is a function of the signed distance $(\phi)$ to the interface. The region exterior to the thrombus $(\phi>0)$ is pure fluid $(\varepsilon=1)$ while within the thrombus $(\phi<0) \varepsilon=0.75$ (as dictated by Diamond [33]). If the porosity field was set as above, a binary function of the signed distance, then the position of the interface would be grid dependent. In this work, the transition from pure fluid to thrombus is smeared across three computational cells, which ensures that the iso-surface of $\varepsilon=0.875$ lies on the thrombus surface (zero-level set).

The rate at which a thrombus forms in vivo is a complex function of the underlying biochemistry, cell signalling and flow conditions. In order to demonstrate the capability of this framework, we focus upon the modulation of thrombus growth through shear stress alone. In order to investigate the efficacy of considering shear stress alone, it will be necessary to compare in silico simulations with in vivo data. In the absence of experimental data, we postulate three scenarios (Table 1).

As the velocity is applicable only at the interface in order to maintain the signed distance property, it is necessary to extend the velocity off the interface. The signed distance function is sampled on a uniform, structured grid, whereas the flow mesh consists of unstructured polyhedral elements. The interface velocity calculated into the flow domain is
Table 1. Thrombus surface normal velocity for three scenarios

\begin{tabular}{ll}
\hline Case I & $V_{\text {normal }}=4.0 \times 10^{-7} \mathrm{~ms}^{-1}$ \\
Case II & $V_{\text {normal }}=4.0 \times 10^{-7} \cdot \tau \mathrm{ms}^{-1}$ \\
Case III & $V_{\text {normal }}=4.0 \times 10^{-7} \cdot \tau^{-1} \mathrm{~ms}^{-1}$ \\
\hline
\end{tabular}

interpolated onto the structured level-set grid, while the signed distance is interpolated onto the flow mesh.

\section{Results}

Computational simulation results are in need of validation and self-consistency checks. Although, owing to space considerations, this will not be expanded, however, it must be mentioned, that for the latter, detailed discretisation-dependence tests have been conducted for all the studies presented; the results shown are invariably obtained using levels of mesh refinement that ensure grid independence. Depending on the problem, this sometimes implies using grids of tens of millions of elements, as shall be discussed in the sequel. For the former, several comparisons with in vitro or in vivo results have been conducted; for example, a recent validation test was conducted that involved detailed laser velocimetry for anatomically accurate aneurysm haemodynamics [34].

\section{Growth and remodelling}

In this example, we explicitly link degeneration of elastin to deviations of WSS from homeostatic value. If the maximum value of WSS during the cardiac cycle is greater or equal to $2 \mathrm{~Pa}$, no elastin degradation occurs, but if the WSS is lower than $0.5 \mathrm{~Pa}$ maximum degeneration occurs, i.e. $75 \%$ of the elastinous constituents at a particular spatial position in the tissue degrade per year. For WSS magnitudes between $0.5 \mathrm{~Pa}$ and $2 \mathrm{~Pa}$, the amount of elastin degraded per year ranges linearly between $75 \%$ per year and $0 \%$ per year. Additionally, in this simulation we confine elastin degradation to occur only in a localised region where the initial out-pouching forms, otherwise the aneurysms continue to enlarge axially along the arterial domain rather than giving rise to well-defined saccular geometries [11]. Figure 2 illustrates the spatial distributions of the elastin concentration and the WSS distributions that act on the endothelial layer of the aneurysm as it evolves. Figure 3 illustrates the evolution of the elastin and collagen strains. Notice that the elastin strains increase significantly, whereas the collagen strains increase negligibly. As the aneurysm enlarges, the continual process of fibre deposition and degradation [35] remodels the natural reference configuration in which collagen fibres are recruited to load bearing, hence the stretch of the collagen fabric is restored to an equilibrium level. Finally, Figure 4 illustrates the evolution of the collagen fibre concentration. The model simulates fibroblasts increasing the collagen content in the arterial wall to compensate for the loss of load bearing owing to the degradation of the elastinous constituents. This enables the aneurysm to stabilise in size. 
Elastin concentration

\begin{tabular}{|l|}
\hline 1 \\
0.9 \\
0.8 \\
0.7 \\
0.6 \\
0.5 \\
0.4 \\
0.3 \\
0.2 \\
\hline 0.1 \\
0.05 \\
0.01 \\
0.005 \\
0.001 \\
\hline
\end{tabular}

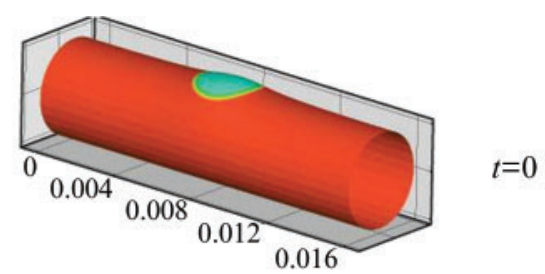

$=0$
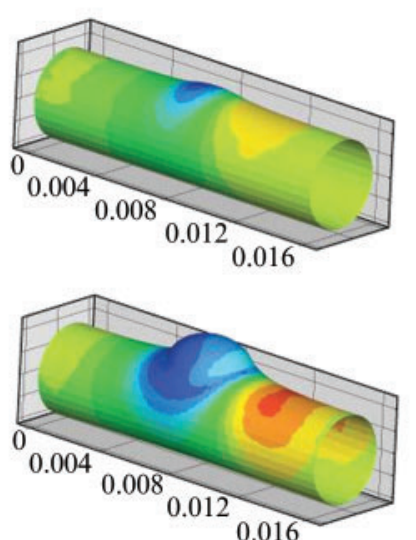

WSS
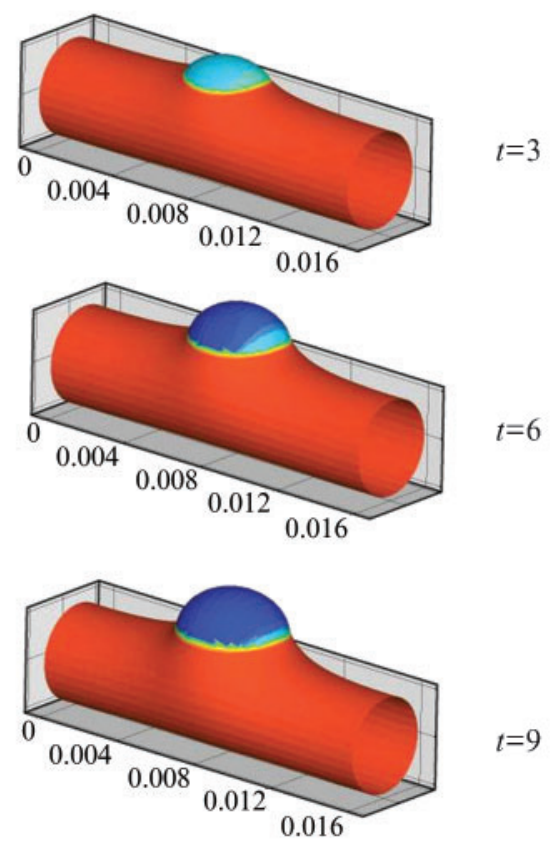

$t=9$
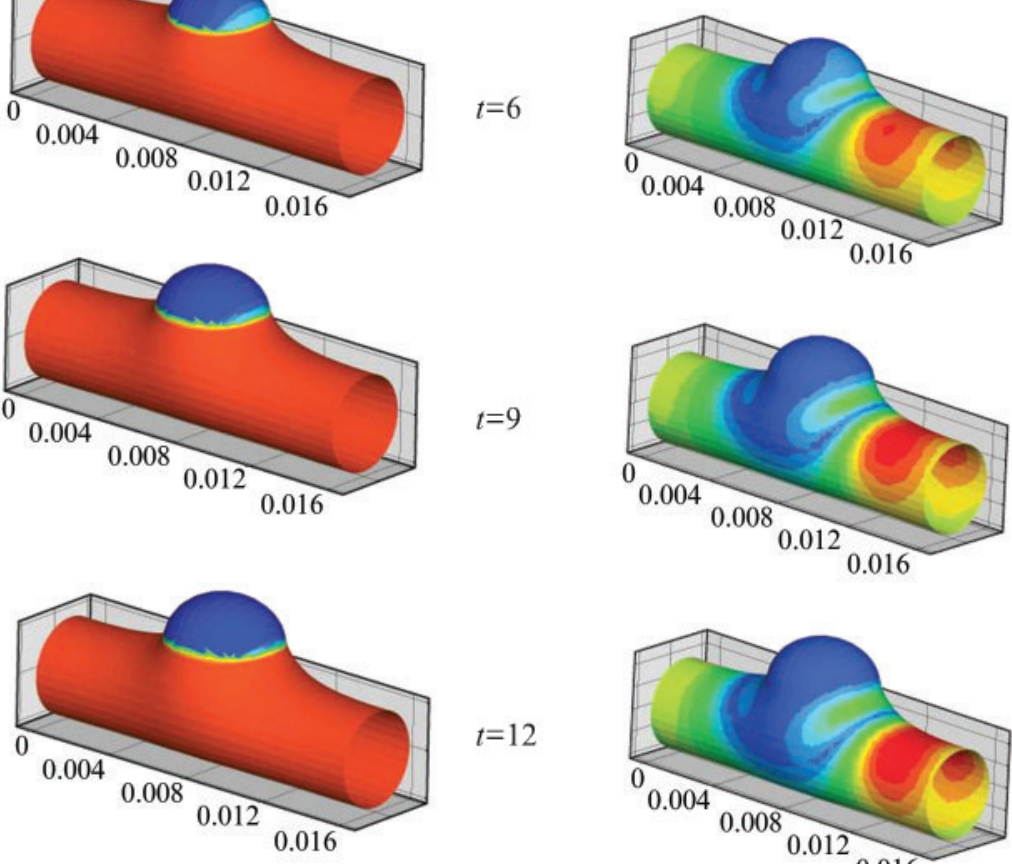

(a)

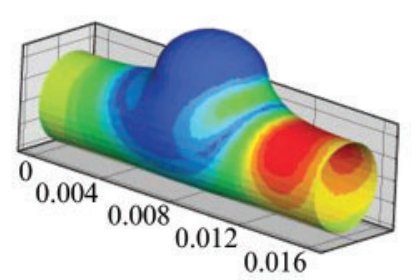

(b)

Figure 2. Computational model of development of a saccular cerebral aneurysm that stabilises in size. The evolution of the elastin concentration (a) and the wall shear stress distribution (b) for $t=0,3,6,9$ and 12 years. Note that in this example all the elastin degrades within the aneurysm dome; also note that there is an asymmetry in the progression of the degradation owing to the asymmetry in the wall shear stress distribution, i.e. it has a greater value at the distal region of the dome.

\section{Interventional modelling}

The case study outlined in the Virtual Intracranial Stenting Challenge (VISC) 2007 [19] is reproduced to demonstrate the potential of CFD-VisCART to model 3D anatomical models of unstented and stented aneurysms. The stereolithographic format files (stl) of the side-wall aneursym and three types of stents geometries used, as well as the flow boundary conditions, are all previously reported in the VISC challenge, and so shall not be discussed further in this article.

In-house comparisons were made between CFDVisCART and an unstructured tetrahedral mesh generated in ICEM (ANSYS ICEM CFD, ANSYS). Qualitatively ICEM gives the user more flexibility, but this results in greater demands for user interaction and hence training; grid generation with VisCART requires less user input.
Between the two meshing techniques, the global results were quantitatively similar at the same mesh densities (data not shown). In this work we present results for the unstented and stented aneurysm, with meshes generated in VisCART. Figure 5 depicts the inflow region by means of an isovelocity surface $\left(v=0.1 \mathrm{~m} \mathrm{~s}^{-1}\right)$ for the unstented model as well as for the aneurysm stented with stent 3 [19]; the benefits of the stent are demonstrated by the clear reduction in the inflow jet when the aneurysm is stented. When evaluating the WSS distribution on the wall of the aneurysms, as shown in Figure 6, the computational modelling also demonstrates the reduction in WSS around the neck of the aneurysm due to stent deployment. In particular, the area of high WSS in the dome of the aneurysm (WSS=1.5 Pa contour plot in Figure 6) is significantly reduced when the stent is deployed. 


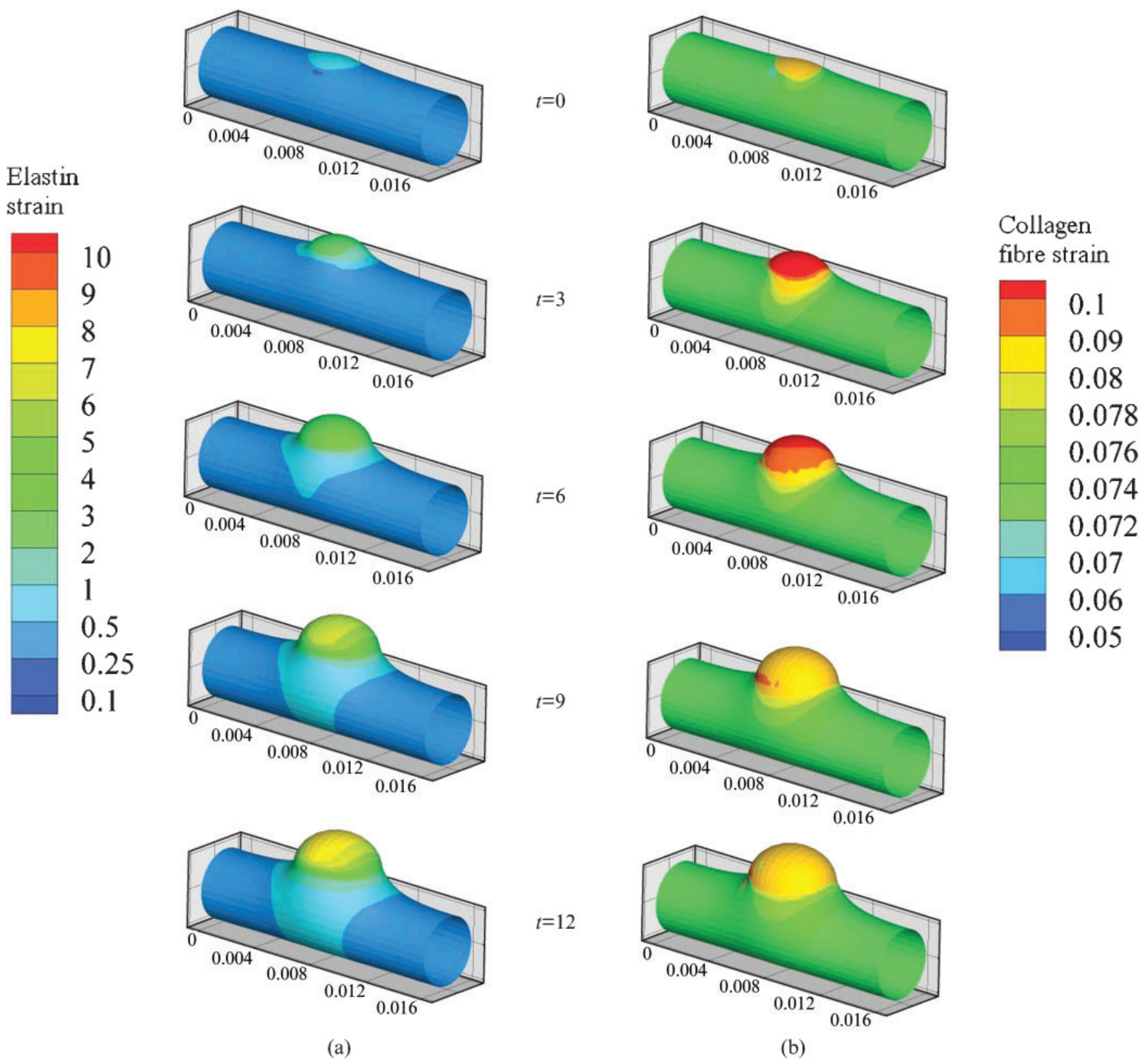

Figure 3. Evolution of the elastin strains (a) and collagen fibre strains (b) for $t=0,3,6,9$ and 12 years. Note that the elastin strains increase significantly (up to 10 ) because of the large deformations of the artery; however, the collagen strains barely increase, i.e. remain around the equilibrium value of 0.073 . This is a consequence of the natural process of fibre deposition and degradation, which is captured by the computational framework, to simulate the remodelling of the collagen fabric.

When evaluating the velocity magnitude contour plot across the neck of the aneurysm, as seen in Figure 7, there is not only a reduction in velocity for all three stents, but also a distinct difference between each stent design, and hence we can now confidently predict the flow reductions induced by different stents in a clinically meaningful framework.

Next, we present results from the haemodynamic modelling of the coil embolisation of a ruptured anterior communicating artery aneurysm, with a large sac of dimensions $11 \times 9.4 \times 7.8 \mathrm{~mm}$, and a neck of $5.2 \mathrm{~mm}$, using the porosity approach. Figure 8 compares velocity magnitude contours of the pre-treatment conditions with those developed after the deployment of the first coil (3.6\% packing by volume and porosity 0.964$)$ and after the completion of the procedure, which occurs with the deployment of 15 coils $(26.5 \%$ packing by volume and porosity 0.735$)$. The assumptions made are non-compliant walls, newtonian behaviour for the blood, with constant viscosity and steady flow. Coiling has a significant effect, even from the very first coil, by changing the velocity profiles, and the gradual introduction of coils inside the aneurysm sac results in a rapid decrease of the velocity up to stagnation, thus providing an ideal haemodynamic environment for thrombus formation. Blood flow is observed at the aneurysm lumen through the coil-mesh interface, even after completing the coiling, which may be a factor in later coil compaction and aneurysm recanalisation that is frequently seen in such wide-necked aneurysms. This is 


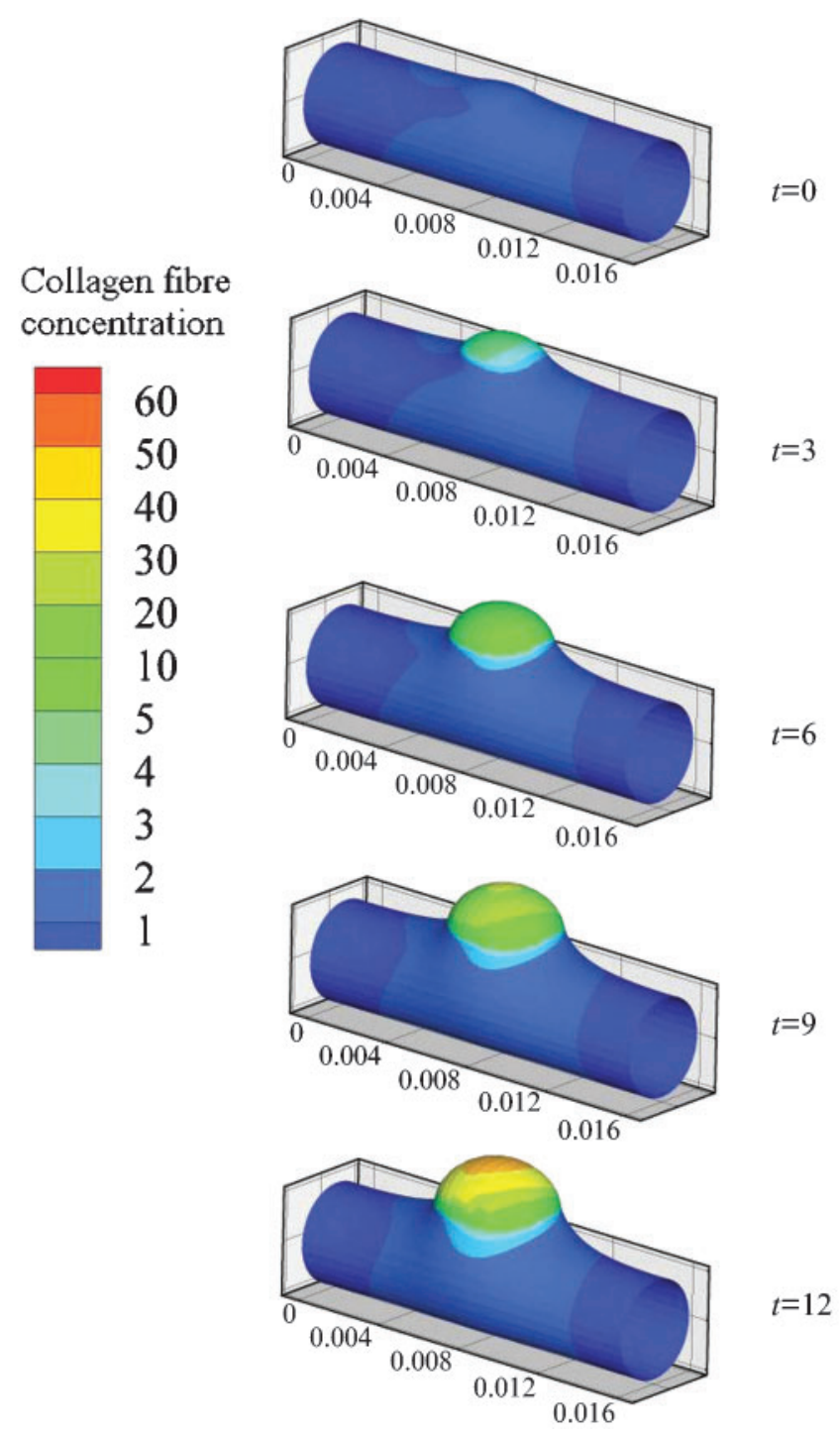

Figure 4. Evolution of collagen fibre concentration. Note that in this example all the elastin degrades within the aneurysm dome; however, the aneurysm stabilises in size (no change in geometry between $t=9$ and $t=12$ ) owing to the collagen fibre concentration increasing to compensate for the loss of elastin. a realistic representation of the clinical situation, as the coil-mesh is not acting as an absolute solid barrier between the aneurysm lumen and the parent vessels, but rather obstructs and gradually slows the intra-aneurysmal blood circulation. Advanced techniques like these can also be used to study further aspects of the coil embolisation method, like the interaction of the system with the microcatheter. For instance, Figure 9 presents intra-aneurysmal blood flow patterns (just before the deployment of the first coil) coupled with the pressure exerted on the catheter for a time instant along the cardiac cycle.

\section{Thrombosis modelling}

As the interface velocity is a function of shear stress, it is difficult to directly compare the same time across the different cases as outlined in Table 1. As an alternative, we highlight the interesting features by choosing timepoints that correspond to similar average propagation distances. For case I the growth is entirely dictated by the initial geometry (see Figure 10a-c), it is interesting to note that the thrombus grows into the daughter vessels prior to full occlusion of the aneurysm lumen. This finding is mirrored in Case 2: relative to growth within the lumen the propagation into the daughter vessel occurs quicker in this scenario. Stipulating that interface velocity is proportional to shear stress sets in place a feedback loop; in regions of high shear the clot grows faster, causing the shear stress to increase and leading to further acceleration of clot progression. In contrast, in Case 3, in which thrombus growth is inhibited by regions of high shear, the propagation of the thrombus into the surrounding vasculature is inhibited.

The thrombus encapsulates many active biological processes; by ignoring these we are assuming that mechanical effects are the dominant driver of thrombus morphology. In reality, the effect of shear stress upon cell-cell interactions is a function of the biochemical state of the cells and, therefore, of time. Post-interventional changes to clot morphology are not uncommon; in this framework temporal changes could be incorporated only by stipulating that the relationship between shear stress and interface velocity

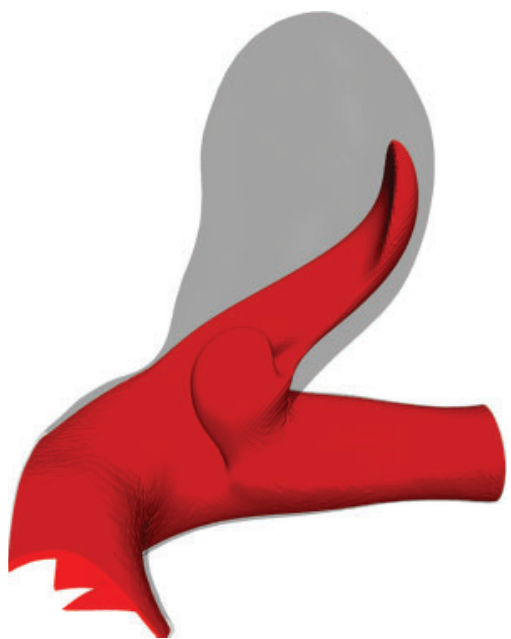

(a)

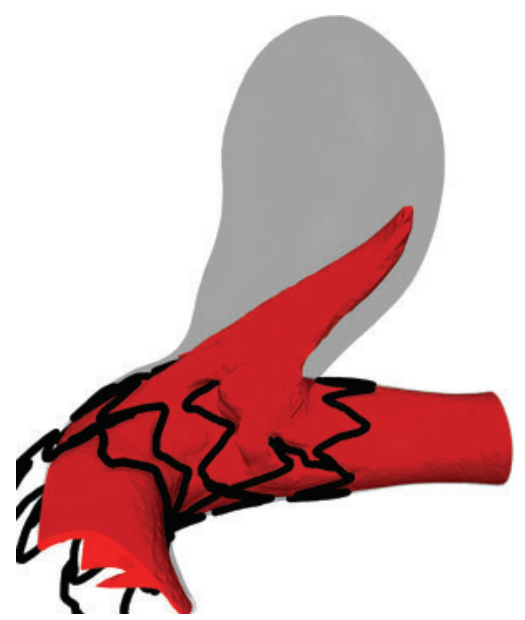

(b)
Figure 5. Inlet flow for an unstented (a) and stented (b) aneurysm: isovelocity surface $v=0.1 \mathrm{~m} \mathrm{~s}^{-1}$. 


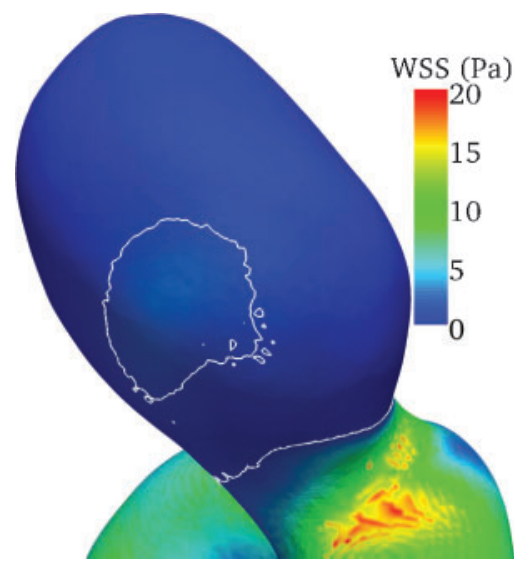

(a)

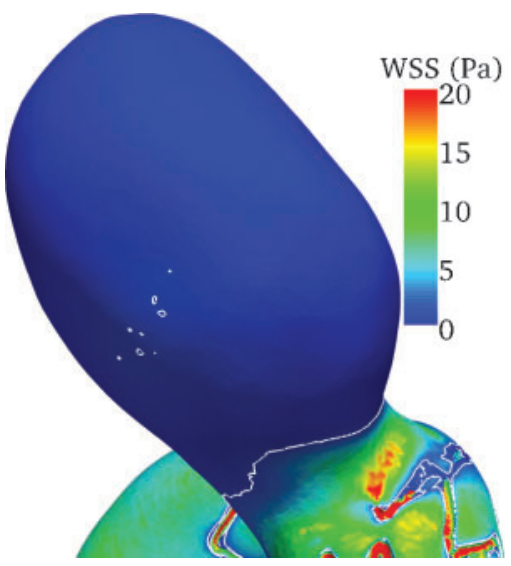

(b)
Figure 6. Wall shear stress (WSS) distribution across the wall of unstented (a) and stented (b) aneurysm. In both cases, white demarcations demonstrates WSS $=1.5 \mathrm{~Pa}$ threshold in the aneurysm dome.

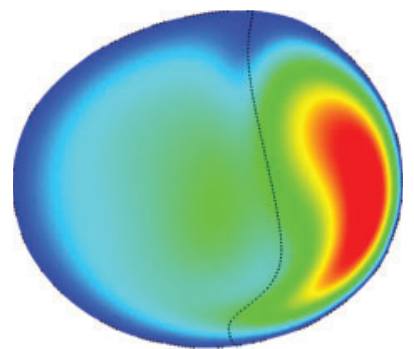

(a)

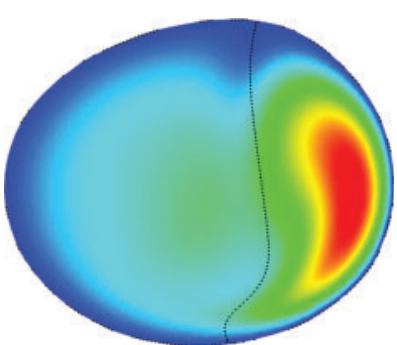

(b)

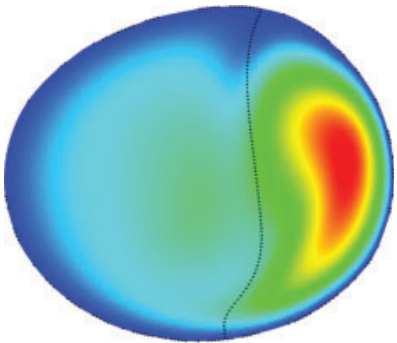

(c)

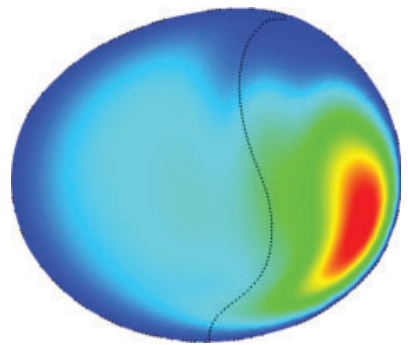

(d)

Figure 7. Velocity magnitude contours for unstented aneurysm (a), stent 1 (b), stent 2 (c) and stent 3 (d).

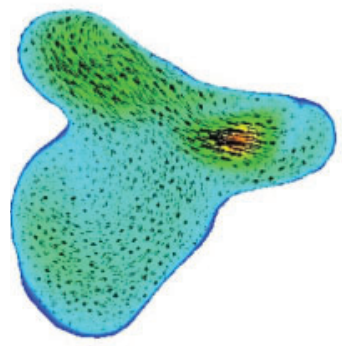

(a)

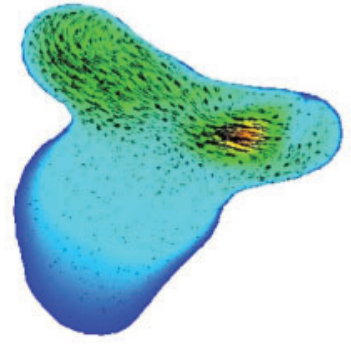

(b)

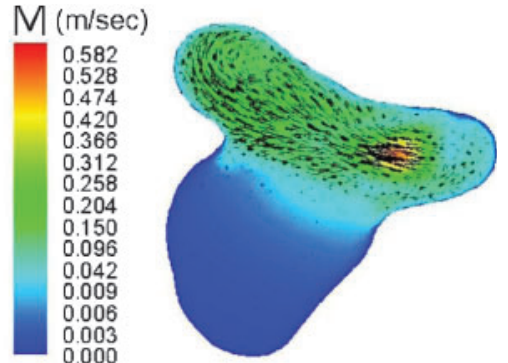

(c)

Figure 8. Velocity magnitude contours $(\mathrm{m} / \mathrm{s}$ ) for coil-free (a) and after the deployment of the first (b) and the fifteenth coil (c) conditions.

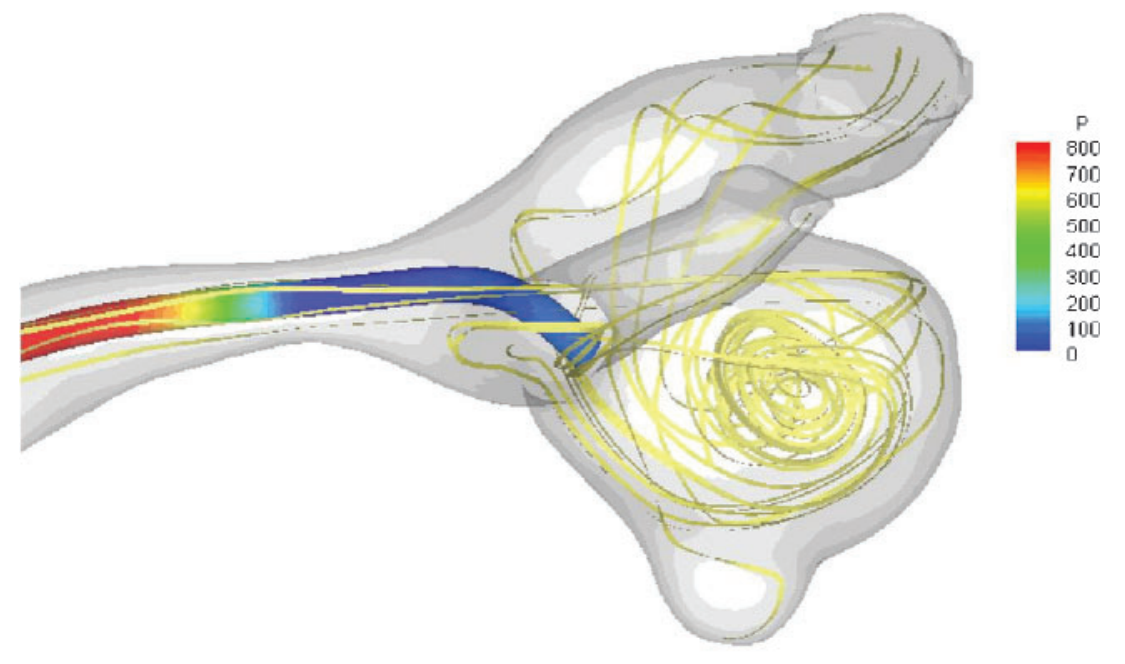

Time $0.0775863 \mathrm{~s}$
Figure 9. Pressure on catheter and flow patterns. 


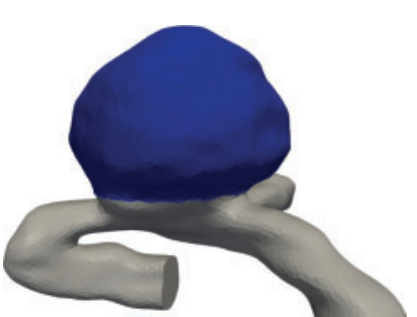

(a)

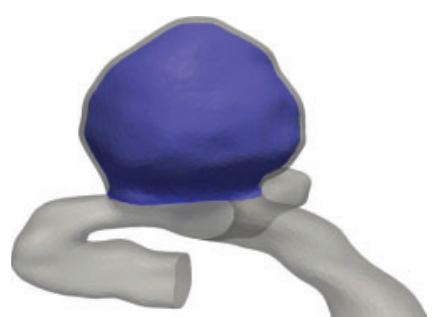

(b)

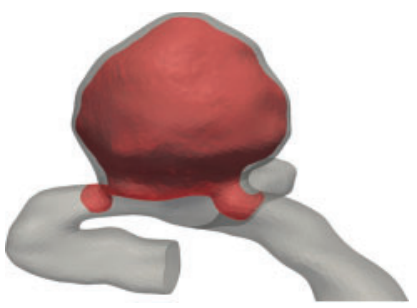

(d)

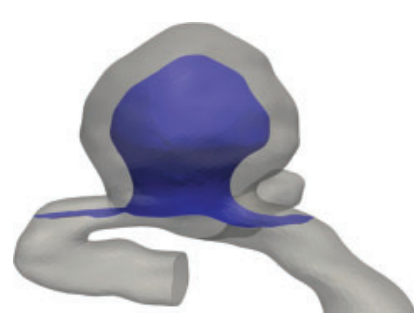

(c)

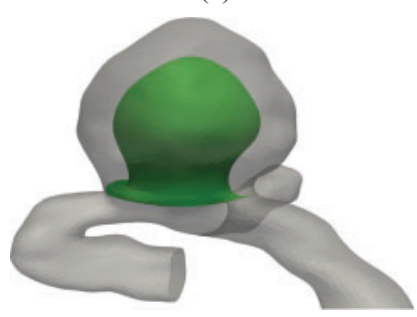

(e)

Figure 10. Clot progression from the wall of a cerebral aneurysm located in close proximity to the middle cerebral artery bifurcation. The thrombus surface is visualised through opaque representation of the vessel wall. Case $1: V_{\text {normal }}=4.0 \times 10^{-7},(a)$ $\mathrm{t}=0$, (b) $\mathrm{t}=400 \mathrm{~s}$, (c) $\mathrm{t}=1600 \mathrm{~s}$. Case $2: \mathrm{V}_{\text {normal }}=4.0 \times 10^{-7} \cdot \tau$, (d) $\mathrm{t}=80 \mathrm{~s}$. Case 3 : $\mathrm{V}_{\text {normal }}=4.0 \times 10^{-7} \cdot \tau^{-1}$, (e) $\mathrm{t}=8500 \mathrm{~s}$.

is also a function of time. To fully understand and optimise clot growth within cerebral aneurysms, it will be necessary to incorporate the competing balance of growth derived from biological signalling and the inhibitory effect of mechanical conditions upon the surface of the thrombus. The future aims for this work are to include basic models of the coagulation cascade and to couple clot growth to other stimuli, such as thrombin and fibrin concentration.

\section{Conclusions}

This paper, has presented a collection of computational techniques aimed at demonstrating the capacity that such tools exhibit in different areas of cerebral aneurysm healthcare planning. We have shown that wall-remodelling techniques can mimic the growth and stabilisation/rupture of aneurysms, and can thus be used as an evaluating tool for haemorrhagic risk. Further, it has been shown that computational techniques of this type can be used to evaluate the capacity of endovascular devices (stents and coils) to treat specific aneurysms. This class of technique can account for both device and lesion variability and also for complex biochemical processes such as thrombosis.

\section{Acknowledgments}

PNW, PES, YV and JVB are grateful to ANEURIST (EU Framework Programme VI) for support. TJB gratefully acknowledges the support of an EPSRC CNA scholarship. ECH gratefully acknowledges the support of an EPSRC DTA Studentship.

\section{References}

1. van Munster CEP, von und zu Fraunberg M, Rinkel GJE, Rinne J, Koivisto T, Ronkainen A. Differences in aneurysm and patient characteristics between cohorts of Finnish and Dutch patients with subarachnoid hemorrhage: time trends between 1986 and 2005. Stroke 2008;39:3166-71,
2. Rinkel GRF, Djibuti M, Algra A, van Gijn J. Prevalence and risk of rupture of intracranial aneurysms: a systematic review. Stroke 1998;29:251-6.

3. Juvela S, Porras M, Poussa K. Natural history of unruptured intracranial aneurysms: probability of and risk factors for aneurysm rupture. J Neurosurg 2000;93:379-87.

4. Wiebers D, Whisnant JP, Huston J, Meissner I, Brown RD, Piepgras DE, et al. Unruptured intracranial aneurysms: natural history, clinical outcome and risks of surgical and endovascular treatment. Lancet 2003;362:103-10,

5. Clarke M. Systematic review of reviews of risk factors for intracranial aneurysms. Neuroradiology 2008;50:653-64.

6. Lu J, Zhou X, Raghavan M. Inverse method of stress analysis for cerebral aneurysms. Biomech Model Mechanobiol 2008;7:477-86.

7. Chatziprodromou I, Tricoli A, Poulikakos D, Ventikos Y. Haemodynamics and wall remodelling of a growing cerebral aneurysm: a computational model. J Biomech 2007;40:412-26.

8. Chatziprodromou I, Poulikakos D, Ventikos Y. On the influence of variation in haemodynamic conditions on the generation and growth of cerebral aneurysms and atherogenesis: A computational model. J Biomech 2007;40:3626-40.

9. Kroon M, Holzapfel GA. A model for saccular cerebral aneurysm growth by collagen fibre remodelling. J Theor Biol 2007;247:775-87.

10. Watton PN, Raberger NB, Ventikos Y. Modelling growth of aneurysms, SPIE Newsroom 2008; doi: 10.1117/2.1200801. 0999.

11. Watton PN, Raberger NB, Holzapfel GA, Ventikos Y. Coupling the haemodynamic environment to the growth of cerebral aneurysms: computational framework and numerical examples. J Biomech Eng 2009;131:101003.

12. Jou LD, Quick CM, Young WL, Lawton MT, Higashida R, Martin A, et al. Computational approach to quantifying haemodynamic forces in giant cerebral aneurysms. AJNR Am J Neuroradiol 2003;24:1804-10.

13. Hassan T, Ezura M, Timofeev EV, Tominaga T, Saito T, Takahashi A, et al. Computational simulation of therapeutic parent artery occlusion to treat giant vertebrobasilar aneurysm. AJNR Am J Neuroradiol 2004;25:63-8.

14. Groden C, Laudan J, Gatchell S, Zeumer H Three-dimensional pulsatile flow simulation before and after endovascular coil embolization of a terminal cerebral aneurysm. Cereb Blood Flow Metab 2001;21:1464-71. 
15. Byun HS, Rhee K. CFD modelling of blood flow following embolisation of aneurysms. Med Eng Phys 2004;26:755-61.

16. Aenis M, Stancampiano AP, Wakhloo AK, Lieber BB. Modeling of flow in a straight stented and nonstented side wall aneurysm model. J Biomed Eng 1997;119:206-12.

17. Stuhne GR, Steinman DA. Finite-element modeling of the hemodynamics of stented aneurysms. J Biomed Eng 2004;126:382-7.

18. Cebral JR, Lohner R. Efficient simulation of blood flow past complex endovascualr devices using an adaptive embedding technique. IEEE T Med Imaging 2005;24:468-76.

19. Radaelli AG, Augsburger L, Cebral JR, Ohta M, Rufenacht DA, Balossina R, et al. Reproducibility of haemodynamical simulations in a subject-specific stented aneurysm model a report on the Virtual Intracranial Stenting Challenge 2007. J Biomech 2008;41:2069-81.

20. Macewen J. The surgical treatment of aortic aneurism. Report on a case successfully treated by production of white thrombus. Ann Surg 1912;56:675-80.

21. Kirchhof K, Welzel T, Mecke C, Zoubaa S, Sartor K. Differentiation of white, mixed, and red thrombi: value of CT in estimation of the prognosis of thrombolysis phantom study. Radiology 2003;228:126-30.

22. Furie B, Furie BC. Mechanisms of disease: Mechanisms of thrombus formation. N Engl J Med 2008;359:938.

23. Hockin MF, Jones KC, Everse SJ, Mann KG. A model for the stoichiometric regulation of blood coagulation. J Biol Chem 2002;277:18322-33.

24. Panteleev MA, Ovanesov MV, Kireev DA, Shibeko AM, Sinauridze EI, Ananyeva NM, et al. Spatial propagation and localization of blood coagulation are regulated by intrinsic and protein c pathways, respectively. Biophys J 2006;90:1489-500.

25. Fogelson AL, Tania N. Coagulation under flow: the influence of flow-mediated transport on the initiation and inhibition of coagulation. Pathophysiol Haemost Thromb 2005;34:91-108.

26. Guy RD, Fogelson AL, Keener JP. Fibrin gel formation in a shear flow. Math Med Biol 2007;24:111-30.

27. Holzapfel GA, Gasser TC, Ogden RW. A new constitutive framework for arterial wall mechanics and a comparative study of material models. J Elast 2000;61:1-48.

28. Watton PN, Hill NA, Heil M. A mathematical model for the growth of the abdominal aortic aneurysm. Biomech Model Mechanobiol 2004;3:98-113.

29. Watton PN, Hill NA. Evolving mechanical properties of a model of abdominal aortic aneurysm. Biomech Model Mechanobiol 2009;8:25-42.

30. Wang ZJ, Srinivasan K. An adaptive Cartesian grid generation method for 'dirty' geometry. Int J Numer Methods Fluids 2002;39:703-17.

31. Kakalis NMP, Mitsos AP, Byrne JV, Ventikos Y. The haemodynamics of endovascular treatment: A computational modelling approach for estimating the influence of multiple coil deployment. IEEE T Med Imaging 2008;27: 814-24.

32. Koponen A, Kataja M, Timonen J. Permeability and effective porosity of porous media. Phys Rev E 1997;56: 3319-25.

33. Diamond SL. Engineering design of optimal strategies for blood clot dissolution. Annu Rev Biomed Eng 1999;1: 427-62.

34. Boutsianis E, Gualal M, Olgac U, Wildermuth S, Hoyer K, Ventikos Y, et al. CFD and PTV steady flow investigation in an anatomically accurate abdominal aortic aneurysm. J Biomech Eng 2009;131:011008.

35. Watton PN, Ventikos Y, Holzapfel GA. Modelling growth and stabilisation of cerebral aneurysms. Math Med Biol 2009;26:133-64. 